\title{
Symmetric Color Ratio in Spiral Architecture
}

\author{
Wenjing Jia, Huaifeng Zhang, Xiangjian He, and Qiang Wu \\ Faculty of Information Technology, University of Technology, Sydney, \\ PO Box 123, Broadway, NSW, Sydney, Australia, \\ \{wejia, hfzhang, sean, wuq\}@it.uts.edu.au
}

\begin{abstract}
Color ratio gradient (CRG) is a robust method used for color image retrieval and object recognition. It has been proven to be illumination-independent and geometry-insensitive when tested on scenery images. However, the color ratio gradient produces unsatisfying matching results when dealing with an object which appears rotated by a certain relative angle in the model and target images. In this paper, we adopt the idea of color ratio gradient and develop a new method called Symmetric Color Ratio (SCR) based on a hexagonal image structure, the Spiral Architecture (SA). We focus on license plate images and our aim is to achieve a higher matching rate between the SCR histogram of the images within same class in order to separate different classes of images. Our experimental results demonstrate that the proposed SCR is robust to changes over view angles.
\end{abstract}

\section{Introduction}

Using color histograms as a stable object representation over change in view for object recognition was first explored by Swain and Ballard [1][2] who introduced the color indexing technique to efficiently recognize objects by matching their color-space histograms. This method, however, did not address the issue of illumination variation. Funt and Finlayson [3] produced a new measurement based on the ratio of color RGB triples in neighboring area to locate objects. Compared with Swain's way, this method is more robust to illumination variation. Other improved methods include illumination-independent color reflection ratios proposed by Nayar and Bolle [4]. Gevers [5][6] further developed the color ratio gradient (CRG) to make it insensitive to the geometry and position of the object, shadows, illuminations, and other imaging conditions.

However, such color ratio gradients suffer the following limitation. When dealing with an object which appears rotated by a certain angle in the target image with respect to the model image, the color ratio gradient produces unsatisfying matching results.

In this paper, we adopt the idea of color ratio gradient and develop a new method called Symmetric Color Ratio (SCR), which is based on a hexagonal image structure, the Spiral Architecture (SA) [7]. By taking use of the higher symmetry of the hexagon, as well as the consistent definition of distance between the central pixels and any of its 6 directly-connected neighbors on hexagon-based image structure [8], a completely symmetric operator has been defined. 
We focus on vehicle license plate images and our aim is to achieve a higher matching rate between the SCR histograms of the images that belong to the same class. We say two license plate images belong to the same class when they have similar foreground and background colors, but they may have quite different contents (characters), size and even view angles. The SCR histogram of license plate images has been created and used as a robust feature to separate different classes of images. Our experimental results demonstrate that the proposed SCR histogram is robust to changes over view angles.

The remaining parts of this paper are organized as follows. The color ratio gradient as a feature for object recognition is firstly summarized in Sect. 2. The Spiral Architecture, on which the proposed SCR is implemented, is briefly introduced in Sect. 3. In Sect. 4, the proposed Symmetric Color Ratio is defined in detail, and the similarity measurement is obtained. In Sect. 5, the proposed algorithm is tested. Conclusions are given in Sect. 6 .

\section{Color Ratio Gradients}

Supposing the sensor response is measured on an infinitesimal surface patch of an inhomogeneous dielectric object and the spectral power distribution of illumination is unknown, the body reflection term at location $\boldsymbol{x}$ in dichromatic reflection model with narrow-band filtering can be written as [5][6],

$$
C_{k}(\boldsymbol{x})=G_{B}(\boldsymbol{x}, \boldsymbol{n}, \boldsymbol{s}) E\left(\boldsymbol{x}, \lambda_{k}\right) B\left(\boldsymbol{x}, \lambda_{k}\right)
$$

where $G_{B}(\boldsymbol{x}, \boldsymbol{n}, \boldsymbol{s})$ is the geometric term dependent on the surface orientation $\boldsymbol{n}$ and illumination direction $\boldsymbol{s}, E\left(\boldsymbol{x}, \lambda_{k}\right)$ is the illumination intensity at light wavelength $\lambda_{k}$, and $B\left(\boldsymbol{x}, \lambda_{k}\right)$ is the surface albedo at light wavelength $\lambda_{k}$.

Gevers proposed the following color constant color ratio[5][6],

$$
M\left(C_{1}^{\boldsymbol{x}_{1}}, C_{1}^{\boldsymbol{x}_{2}}, C_{2}^{\boldsymbol{x}_{1}}, C_{2}^{\boldsymbol{x}_{2}}\right)=\frac{C_{1}^{\boldsymbol{x}_{1}} C_{2}^{\boldsymbol{x}_{2}}-C_{1}^{\boldsymbol{x}_{2}} C_{2}^{\boldsymbol{x}_{1}}}{C_{1}^{\boldsymbol{x}_{2}} C_{2}^{\boldsymbol{x}_{1}}+C_{1}^{\boldsymbol{x}_{1}} C_{2}^{\boldsymbol{x}_{2}}} \quad C_{1} \neq C_{2}
$$

expressing the color ratio between two adjacent image pixels at location $\boldsymbol{x}_{1}$ and $\boldsymbol{x}_{2}$ under two different light wavelengths. It can be seen that $M \in[-1,1]$.

Note that in an infinitesimal area it may be assumed that $G_{B}\left(\boldsymbol{x}_{1}, \boldsymbol{n}, \boldsymbol{s}\right)=$ $G_{B}\left(\boldsymbol{x}_{2}, \boldsymbol{n}, \boldsymbol{s}\right), E\left(\boldsymbol{x}_{1}, \lambda_{C_{2}}\right)=E\left(\boldsymbol{x}_{2}, \lambda_{C_{2}}\right)$, and $E\left(\boldsymbol{x}_{1}, \lambda_{C_{1}}\right)=E\left(\boldsymbol{x}_{2}, \lambda_{C_{1}}\right)$ [5]. By substituting (1) into (2) and factoring out dependencies on object geometry and illumination direction, we have,

$$
\begin{aligned}
M\left(C_{1}^{\boldsymbol{x}_{1}}, C_{1}^{\boldsymbol{x}_{2}}, C_{2}^{\boldsymbol{x}_{1}}, C_{2}^{\boldsymbol{x}_{2}}\right) & =\frac{C_{1}^{\boldsymbol{x}_{1}} C_{2}^{\boldsymbol{x}_{2}}-C_{1}^{\boldsymbol{x}_{2}} C_{2}^{\boldsymbol{x}_{1}}}{C_{1}^{\boldsymbol{x}_{2}} C_{2}^{\boldsymbol{x}_{1}}+C_{1}^{\boldsymbol{x}_{1}} C_{2}^{\boldsymbol{x}_{2}}} \\
& =\frac{B\left(\boldsymbol{x}_{1}, \lambda_{C_{1}}\right) B\left(\boldsymbol{x}_{2}, \lambda_{C_{2}}\right)-B\left(\boldsymbol{x}_{2}, \lambda_{C_{1}}\right) B\left(\boldsymbol{x}_{1}, \lambda_{C_{2}}\right)}{B\left(\boldsymbol{x}_{2}, \lambda_{C_{1}}\right) B\left(\boldsymbol{x}_{1}, \lambda_{C_{2}}\right)+B\left(\boldsymbol{x}_{1}, \lambda_{C_{1}}\right) B\left(\boldsymbol{x}_{2}, \lambda_{C_{2}}\right)}(3)
\end{aligned}
$$

It is seen that color ratio is independent of light intensity, color, viewing condition, and object geometry characteristic. It is determined by the ratio of surface albedo only [5]. 


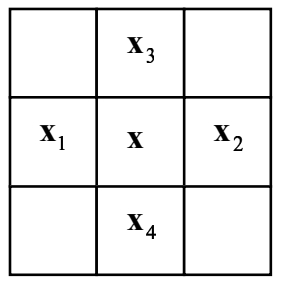

Fig. 1. Locations of four neighbors that are involved in the computation of the color ratio gradient at the central pixel $\boldsymbol{x}$.

Gevers also defined the gradient of the color constant color ratio as [5][6],

$$
\begin{aligned}
\nabla M\left(C_{1}^{\boldsymbol{x}_{1}}, C_{1}^{\boldsymbol{x}_{2}}, C_{2}^{\boldsymbol{x}_{1}}, C_{2}^{\boldsymbol{x}_{2}}\right)= & \left(M\left(C_{1}^{(x-1, y)}, C_{1}^{(x+1, y)}, C_{2}^{(x-1, y)}, C_{2}^{(x+1, y)}\right)^{2}\right. \\
& \left.+M\left(C_{1}^{(x, y-1)}, C_{1}^{(x, y+1)}, C_{2}^{(x, y-1)}, C_{2}^{(x, y+1)}\right)^{2}\right)^{\frac{1}{2}}(4)
\end{aligned}
$$

where $(x-1, y),(x+1, y),(x, y-1)$, and $(x, y+1)$ are locations of four adjacent neighbors of $\boldsymbol{x}=(x, y)$, as shown in Fig.1.

On standard RGB color space, the three-channel color ratios can be written as,

$$
\left\{\begin{array}{l}
M\left(R^{\boldsymbol{x}_{1}}, R^{\boldsymbol{x}_{2}}, G^{\boldsymbol{x}_{2}}, G^{\boldsymbol{x}_{1}}\right)=\frac{R^{\boldsymbol{x}_{1}} G^{\boldsymbol{x}_{2}}-R^{\boldsymbol{x}_{2}} G^{\boldsymbol{x}_{1}}}{R^{\boldsymbol{x}_{2}} G^{\boldsymbol{x}_{1}}+R^{\boldsymbol{x}_{1}} G^{\boldsymbol{x}_{2}}} \\
M\left(R^{\boldsymbol{x}_{1}}, R^{\boldsymbol{x}_{2}}, B^{\boldsymbol{x}_{2}}, B^{\boldsymbol{x}_{1}}\right)=\frac{R^{\boldsymbol{x}_{1}} B^{\boldsymbol{x}_{2}}-R^{\boldsymbol{x}_{2}} B^{\boldsymbol{x}_{1}}}{R^{\boldsymbol{x}_{2}} B^{\boldsymbol{x}_{1}}+R^{\boldsymbol{x}_{1}} B^{\boldsymbol{x}_{2}}} \\
M\left(G^{\boldsymbol{x}_{1}}, G^{\boldsymbol{x}_{2}}, B^{\boldsymbol{x}_{2}}, B^{\boldsymbol{x}_{1}}\right)=\frac{G^{\boldsymbol{x}_{1}} B^{\boldsymbol{x}_{2}}-G^{\boldsymbol{x}_{2}} B^{\boldsymbol{x}_{1}}}{G^{\boldsymbol{x}_{2}} B^{\boldsymbol{x}_{1}}+G^{\boldsymbol{x}_{1}} B^{\boldsymbol{x}_{2}}}
\end{array}\right.
$$

Thus, by substituting (5) into (4), three-channel color ratio gradients on RGB space can be easily obtained. Each $\nabla M\left(C_{1}^{\boldsymbol{x}_{1}}, C_{1}^{\boldsymbol{x}_{2}}, C_{2}^{\boldsymbol{x}_{1}}, C_{2}^{\boldsymbol{x}_{2}}\right)$ in (4) can be viewed as being computed in a Quasi-Prewitt operator, as illustrated in Fig.1, where $\boldsymbol{x}$ is the central (current) pixel. It can be seen that respectively two neighbors in two directions, i.e., horizontal and vertical directions, have been involved in the computation of the color ratio gradient in the central pixel located at $\boldsymbol{x}$.

\section{Spiral Architecture}

Color ratio gradient is defined on the conventional square-based image structure. Hexagon-based image structure, however, due to its higher symmetry and consistent distance definition between any two adjacent neighbors, can simplify the algorithm design and has attracted many people to do research on it for more than 40 years [8]. In this project, we take use of the above advantages of the hexagon-based image structure and propose the symmetric color ratio (SCR). 


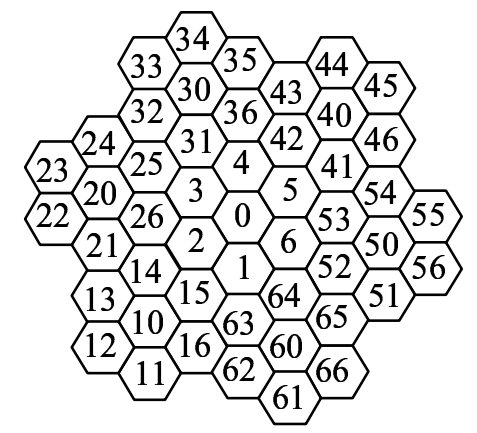

Fig. 2. The Spiral Addressing.

Our SCR is implemented based on a relatively new hexagon image structure, called Spiral Architecture (SA).

The Spiral Architecture (SA) is a unique image representation scheme, proposed by Sheridan [7]. It represents a digital image as a collection of hexagonal pixels, where each hexagonal pixel is addressed in power of seven with a pattern of spiral (see Fig. 2). The hexagon-based image representation and the unique spiral addressing scheme, together with two later proposed mathematic operations, Spiral Addition and Spiral Multiplication, is called Spiral Architecture (SA) [7]. For more details, please refer [7].

Since there is currently no mature hardware device to sample and display images based on hexagonal grids, researchers on hexagonal-based image processing have to use square pixels to mimic hexagonal pixels. Wu et al. [9] constructed a novel mimic scheme called virtual Spiral Architecture, on which images on square grids can be smoothly converted to or from virtual Spiral Architecture in order to test algorithms based on hexagon grids. However, this mimic scheme unavoidably introduces certain loss of resolution of image information which results in blur effects. Fig. 3 gives a pairs of vehicle images which are represented on normal square structure and virtual Spiral Architecture respectively. Imaging area in Spiral Architecture, as shown in Fig.3(b), contains $7^{6}=117649$ hexagonal grids. In order to avoid the blur effects of approximation on the comparison between the experimental results obtained on two different image structures, experiments on square structure are implemented on the virtual SA-processed images to make the results comparable. Also, the number of pixels in the images is calculated as the total number of valid pixels.

\section{Symmetric Color Ratio}

In color ratio gradient (CRG) algorithm, only color ratios between two pixels in horizontal and vertical directions (see Fig.1) are involved into the computation of the final color ratio gradient. Once the objects have rotated with a certain angle relative to the background, however, the color ratio gradient at each pixel 


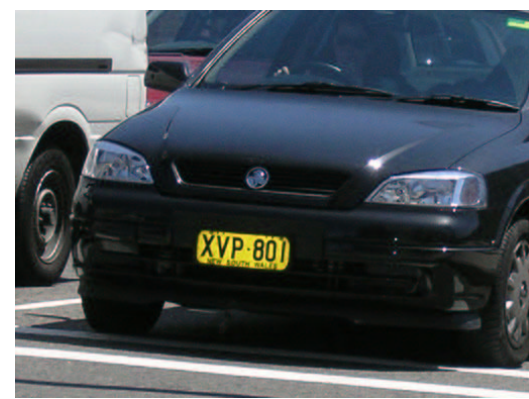

(a)

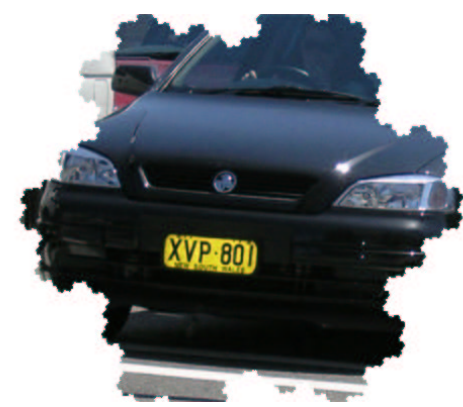

(b)

Fig. 3. (a) Vehicle image and (b) its representation on Spiral Architecture (SA).

will be changed accordingly, and hence the CRG histogram is sensitive to the rotation.

In our method, a symmetric color ratio will be proposed. The contribution of doing so is that a symmetric definition takes into account the contribution of the color changes along three diagonal directions rather than horizontal and vertical directions only. In a local area with a cluster of seven pixels, for example, three directions are able to describe the color changes near the central pixel adequately. Although the similar idea may also possibly be applied in square grids, the inconsistent definition of the distance between the two neighbor pixels in diagonal direction and horizontal/vertical directions in square grids always brings uncertainty about the contribution of each component to the combined value. Hexagon-based algorithm, thanks to its symmetric structure, does not meet such kind of trouble. In our algorithm, the color ratio gradient is computed in a window as shown in Fig.4. The window size should not be chosen too large. Otherwise, it breaches the assumption in equation (3), i.e., calculation must be performed on an infinitesimal surface area. We explain the algorithm in detail as follows.

\subsection{Symmetric Color Ratio in Spiral Architecture}

Let $M_{\boldsymbol{x}}$ denote the color ratio between two horizontal neighbors of a pixel and $M_{\boldsymbol{y}}$ denote the color ratio between two vertical neighbors of a pixel, where the subscript $\boldsymbol{x}$ denotes horizontal direction and $\boldsymbol{x}$ denotes the vertical direction. In the conventional square grids, the definition of the color ratio gradient in (4) can be simplified as:

$$
\nabla M=\sqrt{M_{\boldsymbol{x}}^{2}+M_{\boldsymbol{y}}^{2}}
$$

Following the same naming convention, we use $\nabla M_{S C R}$ to denote the symmetric color ratio of an image at a given reference hexagon point $\boldsymbol{x}$. Without loss of generality, we define $M_{1}, M_{2}$, and $M_{3}$, as shown in Fig.4(a), to denote 


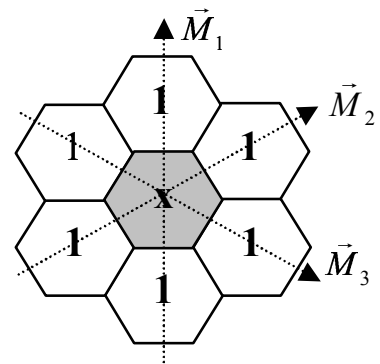

(a)

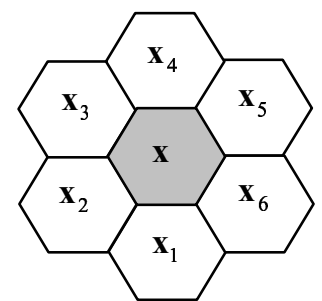

(b)

Fig. 4. (a) Symmetric color ratios $\boldsymbol{M}_{1}, \boldsymbol{M}_{2}$, and $\boldsymbol{M}_{3}$ at the central shadowed hexagonal pixel $\boldsymbol{x}$ in 3 directions. (b)The locations of the six directly connected neighbors of the central hexagonal pixel in Spiral Architecture (SA).

three color ratios in three diagonal directions respectively as:

$$
\left\{\begin{aligned}
M_{1}\left(C_{i}^{\boldsymbol{x}_{1}}, C_{i}^{\boldsymbol{x}_{4}}, C_{j}^{\boldsymbol{x}_{1}}, C_{j}^{\boldsymbol{x}_{4}}\right)=\frac{C_{i}^{\boldsymbol{x}_{1}} C_{j}^{\boldsymbol{x}_{4}}-C_{i}^{\boldsymbol{x}_{4}} C_{j}^{\boldsymbol{x}_{1}}}{C_{i}^{\boldsymbol{x}_{4}} C_{j}^{\boldsymbol{x}_{1}}+C_{i}^{\boldsymbol{x}_{1}} C_{j}^{\boldsymbol{x}_{4}}} \\
M_{2}\left(C_{i}^{\boldsymbol{x}_{2}}, C_{j}^{\boldsymbol{x}_{5}}, C_{i}^{\boldsymbol{x}_{2}}, C_{j}^{\boldsymbol{x}_{5}}\right)=\frac{C_{i}^{\boldsymbol{x}_{2}} C_{j}^{\boldsymbol{x}_{5}}-C_{i}^{\boldsymbol{x}_{5}} C_{j}^{\boldsymbol{x}_{2}}}{C_{i}^{\boldsymbol{x}_{5}} C_{j}^{\boldsymbol{x}_{2}}+C_{i}^{\boldsymbol{x}_{2}} C_{j}^{\boldsymbol{x}_{5}}} \quad i \neq j \\
M_{3}\left(C_{i}^{\boldsymbol{x}_{3}}, C_{j}^{\boldsymbol{x}_{6}}, C_{i}^{\boldsymbol{x}_{3}}, C_{j}^{\boldsymbol{x}_{6}}\right)=\frac{C_{i}^{\boldsymbol{x}_{3}} C_{j}^{\boldsymbol{x}_{6}}-C_{i}^{\boldsymbol{x}_{6}} C_{j}^{\boldsymbol{x}_{3}}}{C_{i}^{\boldsymbol{x}_{6}} C_{j}^{\boldsymbol{x}_{3}}+C_{i}^{\boldsymbol{x}_{3}} C_{j}^{\boldsymbol{x}_{6}}}
\end{aligned}\right.
$$

where $\left\{\boldsymbol{x}_{i}\right\}_{i=1,2, \cdots, 6}$ are the locations of six neighbors of the point $\boldsymbol{x}$. The locations of six neighbors of a current (central) pixel are illustrated in Fig.4(b).

Thus we have the Symmetric Color Ratio (SCR) defined as:

$$
\nabla M_{S C R}=\sqrt{M_{1}^{2}+M_{2}^{2}+M_{3}^{2}}
$$

In Spiral Architecture, since the distance between the central point and any of its six neighboring points is identical, the resulted symmetric color ratio $\nabla M_{S C R}$ is symmetric in three directions rather than two directions, and thus less sensitive to the rotation.

\subsection{Similarity Measure}

A similarity function is needed to return a numerical measure of similarity between the model and target images. In this paper, we use the histogram of the proposed symmetric color ratio (SCR) as a feature of the model and target images in order to numerically measure the similarity between each other. The advantage of using histogram is the robustness to geometric changes of projected objects.

A three-dimensional SCR histogram $\boldsymbol{H}$ is created and chosen as the measurement to compare the similarity between the SCR histograms of two images. 
The three axis of SCR histogram $\boldsymbol{H}$ represent values of SCR between $R$ and $G$ components, simplified as $\nabla M_{R G}$, between $R$ and $B$ components, simplified as $\nabla M_{R B}$, and between $G$ and $B$ components, simplified as $\nabla M_{G B}$ respectively. The value $h(i, j, k)$ of each unit in the histogram $\boldsymbol{H}$ denotes the total number of frequencies of which $\nabla M_{R G}, \nabla M_{R B}$, and $\nabla M_{G B}$ take values of $i, j$, and $k$ respectively.

In order to make such matching invariant to the dimension of image, the created histogram is normalized by the total number of pixels in the image. By such a way, the object matching problem is converted to a simple problem that, to what extent the SCR histogram created for the model image is like the SCR histogram created for the target image.

One straightforward method to calculate the matching rate between two histograms is histogram intersection [2].

Assume the SCR histograms of the model image and target image are $\boldsymbol{H}_{M d l}$ and $\boldsymbol{H}_{T g t}$ respectively, the histogram intersection between the pair of histograms can be defined as:

$$
\boldsymbol{H}_{M d l} \cap \boldsymbol{H}_{T g t}=\frac{\sum_{i, j, k=1}^{n} \min \left(h_{M d l}(i, j, k), h_{T g t}(i, j, k)\right)}{\sum_{i, j, k=1}^{n} h_{M d l}(i, j, k)}
$$

where $n$ denotes the dimension (bin size) of each axis. It can be seen from (7) and (8) that $\nabla M_{S C R} \in[0, \sqrt{3}]$. In this paper, we take identical bin sizes $n=100$ for three axis, i.e., $\nabla M_{S C R}$ are normalized into the range of $[0,100]$ for the convenience of computation.

When both $\boldsymbol{H}_{M d l}$ and $\boldsymbol{H}_{T g t}$ are normalized properly, i.e., $\sum_{i, j, k=1}^{n} h(i, j, k)=$ 1, (9) can be simplified as:

$$
\boldsymbol{H}_{M d l} \cap \boldsymbol{H}_{T g t}=\sum_{i, j, k=1}^{n} \min \left(h_{M d l}(i, j, k), h_{T g t}(i, j, k)\right)
$$

A higher histogram matching rate indicates that a better matching between the SCR histograms of model and target images. Higher matching rates are expected when the model image and the target images are within the same class, or when they have similar foreground and background colors, but may have quite different content, size and even view angle.

\section{Experimental Results}

In this project, we focus on license plate images and our aim is to achieve a higher matching rate between the SCR histograms of two license plate images that are taken from the same class.

The experiments are finished in three parts. In Sect. 5.1, the performance of the proposed SCR histogram is evaluated where SCR is taken as a feature to separate license plate images that belong to different classes. In Sect. 5.2, the independence of SCR on model images which contain quite different characters is proved. In Sect. 5.3, the SCR histogram is shown insensitive to the model images which appear to have a rotation angle. The details are explained as follows. 


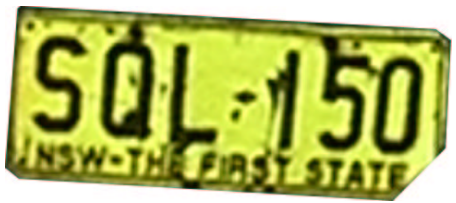

(a)

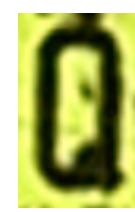

(b)

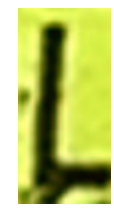

(c)

Fig. 5. An example of cutting a small part of a license plate image(a) to form model $\operatorname{images}(\mathrm{b})(\mathrm{c})$.

\subsection{Similarity Measurement}

Matching rates between the model image and the target images within same class are expected to be relatively high, while they should be very low between different classes of images.

In this experiment, without loss of generality, two classes of vehicle license plate images are tested, namely, license plates with yellow background and license plates with white background. We say two license plate images belong to the same class when they have similar foreground and background colors, but they may have quite different content (characters), size and viewing conditions. For each class, we randomly select a license plate image and cut a small part of image from it (see for example Fig.5.) as the model image of this class. Then, the SCR histogram of the model image is computed and matched with the SCR histograms of the various license plate images (target image) within same class and from another class.

The selection of the model image is quasi-random. Any part of the license plates that contains at least one complete character can be chosen as a model image. Obviously, the larger the size of the model image is, the longer processing time will be needed.

The experiment is done on 64 yellow plates and 27 white plates with different characters, sizes, orientations and illumination conditions. According to our experiments, an average matching rate of $83.5 \%$ within the same class can be obtained, while the average matching rate is $44.4 \%$ for two images that are taken from different classes. This demonstrates that the SCR histogram can be used as robust feature to separate the different classes of license plate images easily.

\subsection{Insensitiveness to the Content of Model Images}

License plates in Australia may contain characters including twenty-six capital letters $A \sim Z$ and ten Arabic digits $0 \sim 9$. As we mentioned in previous subsection, the model image should be selected quasi-randomly. This is to say, no matter which character has been included in the model image, the similarity measurement, i.e., the similarity between the model image and the target images, should be very high and stable.

In this experiment, the model image is still chosen from cutting at least one complete character from the license plate images. However, we cut different parts 


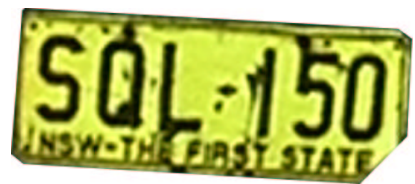

(a)

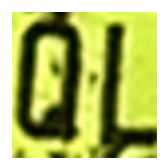

(b)

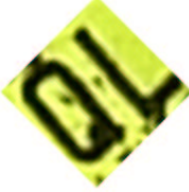

(c)

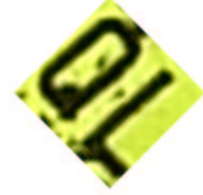

(d)

Fig. 6. Experiments-3: Similarity measurement with rotated model images: an example. (a) Target image; (b) model image without rotation relative to (a); (c) model image with $45^{\circ}$ rotation relative to (a); (d) model image with $-45^{\circ}$ rotation relative to (a).

of a license plate image which contain different characters as model image to test the matching rate between their SCR histograms. The different characters that we chose have large appearance difference, such as "Q" and "L", of which the former contains more curve edge information, while the latter contains more linear edge information.

The experiments give stable matching rates for most character structures. For example, the matching rates of SCR histogram for the case shown in Fig. 5 using model images that contain "Q" and "L" are $81.6 \%$ and $81.3 \%$ respectively. This explains that the matching result using SCR histogram is insensitive to the content inside the images.

\subsection{Insensitiveness to Object Rotation}

In automatic license plate recognition practice, the vehicle images may be tilted to some extent due to uneven or curvy road surface. As a result, the license plate on vehicle may appear to be rotated with a certain angle to the background, which results that the vertical and horizontal gradient information along edges of characters in license plates will be changed to some extent. However, when $\mathrm{SCR}$ is used, the histogram matching rate should be stable due to the symmetric feature of the algorithm.

In this experiment, we keep the characters of model image and the target images unchanged, but rotate the model image with a certain angle relative to the model image, as shown in Fig. 6, then compute the SCR histogram matching rate respectively and compare them.

The matching rates of SCR histogram between the rotated model image and the target images from the same class remain significantly higher than the matching rates obtained for images from different classes. Moreover, for some character structures, the SCR histogram gives more stable matching rate than CRG histogram. For example, the matching rate between the target image in Fig. 6(a) and model images in Fig. 6(b), (c), and (d) are 87.5\%, 88.4\% and 88.3\% respectively when using SCR histogram for histogram matching. While using CRG histogram, these matching rates are $86.5 \%, 76.2 \%$ and $76.4 \%$ respectively. This demonstrates hat the SCR histogram is less sensitive to the rotation of the objects and further proves the robustness of the proposed SCR histogram. 


\section{Conclusions}

The color ratio gradient algorithm has limitations while applied to objects that appear relatively rotated with a certain angle with respect to the background. In this paper, we adopt the idea of color ratio gradient and develop a Symmetric Color Ratio (SCR), which considers three directions around a central pixel with identical symmetric feature. The SCR histogram is applied to license plate images in order to find a higher similarity measurement between images that belong to same class. Our experimental results show that the SCR histogram is insensitive to different characters, sizes, colors, orientations and illumination conditions when being applied to separate license plate images. Besides, the independence of the algorithm on the model images and the robustness to the changes over rotation angle has also been proven. This demonstrates that the proposed algorithm can be used as a robust feature for license plate images.

\section{Acknowledgement}

The work in this paper is supported by Australian Research Council (ARC) Large Research Grant No. DP0451666.

\section{References}

1. Swain, M.J. and D.H. Ballard, "Indexing via color histograms", Computer Vision 1990. Proceedings, Third International Conference on, 1990: p. 390-393.

2. Swain, M.J. and D.H. Ballard, "Color Indexing", International Journal of Computer Vision, 1991. 7(1): p. 11-32.

3. Funt, B.V. and G.D. Finlayson, "Color constant color indexing", Pattern Analysis and Machine Intelligence, IEEE Transactions on, 1995. 17(5): p. 522-529.

4. Nayar, S.K. and R.M. Bolle, "Reflectance ratio: A photometric invariant for object recognition", Computer Vision, 1993. Proceedings, Fourth International Conference on, 1993: p. 280-285.

5. Gevers, T. and W.M. Smeulders, "Color constant ratio gradients for image segmentation and similarity of texture objects", Computer Vision and Pattern Recognition, 2001. CVPR 2001. Proceedings of the 2001 IEEE Computer Society Conference on, 2001. 1: p. I-18-I-25 vol.1.

6. Gevers, T., "Image segmentation and similarity of color-texture objects", Multimedia, IEEE Transactions on, 2002. 4(4): p. 509-516.

7. P. Sheridan, "Spiral Architecture for machine vision," $\mathrm{PhD}$ Thesis, University of Technology, Sydney, 1996.

8. X. He and W. Jia, "Hexagonal Structure for Intelligent Vision", Proceedings of the 1st International Conference on Information and Communication Technology (ICICT 2005) (IEEE), Karachi, Pakistan, August 2005.

9. Q. Wu, X. He, and T. Hintz, "Virtual Spiral Architecture," Proceedings of the International Conference on Parallel and Distributed Processing Techniques and Applications, vol. 1, pp. 399-405, 2004. 